\section{International Congress of Sexually Transmitted Diseases, 19-22 October 1997}

The city of Seville in Andalucia was host to the 12th meeting of the International Society of Sexually Transmitted Diseases Research (ISSTDR) and to the first joint meeting with the International Union Against Sexually Transmitted Infections (IUSTI). There were over 1000 delegates and more than 80 countries were represented. Several precongress workshops and symposia were held; three were of particular note, two focusing on Chlamydia trachomatis screening and new diagnostic methods, the other highlighting problems faced by the developing world. The themes of transmission and prevention of sexually transmitted infection (STI) formed the cornerstone of the six plenary sessions There were 64 symposia presentations, 187 oral presentations, and 555 poster presentations together with many individual discussions on matters arising.

\section{Chlamydia trachomatis}

The meeting once again highlighted the asymptomatic nature of chlamydial infection and the poor suitability of cell culture for screening purposes. In a satellite symposium Dr R Verkooyen (Netherlands) reported on the superiority of nucleic acid amplification assays for the detection of $C$ trachomatis, with specificities in male first void urine (FVU) exceeding $99 \%$ in all assays and sensitivities of $84 \%$ for ligase chain reaction (LCR) ( $\mathrm{LCx}$ Abbott), $95 \%$ for polymerase chain reaction (PCR) (Cobas Amplicor, Roche), and 85\% for transcription mediated amplification (TMA) (Amplicor C trachomatis, GenProbe) with the additional advantages of automation. However, it was advocated that confirmation should be sought for positive results in a low prevalence area. The presence of endogenous inhibitors in urine, indicated by Dr J Mahony (Canada), can produce false negative results in all assays-for example, $\beta$ HCG and crystals (PCR), nitrites and haemoglobin (LCR), nitrites and crystals (TMA). However, storing at $4^{\circ} \mathrm{C}$, freezing at $-70^{\circ} \mathrm{C}$ or phenol can remove the inhibition in many cases.

The utilisation of DNA quantification from other sites (vulval sweep, vaginal flush) and self sampling techniques by asymptomatic individuals were detailed (abstracts O176, O178, P501, P502).

An eloquent presentation, again in a satellite symposium, by Dr J Paavonen (Finland) speaking on the importance of $C$ trachomatis screening using a decision model of universal screening versus no screening, came to the conclusion that if the prevalence rate in a population was more than $3.9 \%$ the screening would pay for itself with the following predicted health related outcomes; a proportional increase of $62.3 \%$ of cured patients; a drop in infertility and ectopic pregnancy rates by $50 \%$; a reduction in chronic pain by $51 \%$; and a fall in laparotomy rates by $66 \%$.

\section{Neisseria gonorrhoeae}

With the introduction of DNA amplification, non-cultural methods for the detection of $N$ gonorrhoeae were discussed. LCR was shown to be highly specific and more sensitive than pharyngeal and rectal culture in homosexual men (abstract O182). Preliminary data also showed high sensitivity with FVU and vulval smears and may well replace invasive sampling for gonococcal diagnosis (abstract P297). However, there was concern about emerging antimicrobial resistance with several reports presented by members of the Gonococcal Antimicrobial Susceptibility Programme (GASP). This group, sponsored by the WHO, is an international laboratory based surveillance network to monitor the extent and type of antibiotic resistance of $N$ gonorrhoeae (abstract O141).

\section{Pelvic inflammatory disease (PID)}

PID was classed as a global emergency with the prevention of PID being the public health goal. It was felt that knowledge of PID needed to be moved to the bedside for any tangible effect-for example, in a randomised clinical trial absence of vaginal white blood cells on a vaginal wet mount excluded the diagnosis of PID with a $95 \%$ sensitivity and a negative predictive value of $89 \%$ (abstract P457). Dr J Paavonen (Finland) questioned whether the presentation of PID globally was uniform given varying epidemiological features. The difficulty of transposing Western experience to the developing world was highlighted by the overall incidence of infertility which in Nigeria is $53 \%$, with almost $17 \%$ being due to genital tuberculosis. Considering that the clinical diagnosis of PID has a sensitivity of only $60-70 \%$ the use of magnetic resonance imaging in the future may be a more sensitive tool (abstract S35).

The new CDC guidelines for the management of PID were discussed by Dr K A Workowski (USA) which showed that there was consensus for $N$ gonorrhoeae and $C$ trachomatis cover but that the role played by anaerobic infection and its treatment was unclear. Attention was also drawn to the inconsistency in the management of PID and the discrepancy between guideline production and utilisation with a study which indicated that general practitioners in Norway used 63 different treatment regimens for PID when compared with the WHO guidelines. It was felt that more research was needed in this area (abstract S37).

\section{Syphilis}

A prospective study of HIV positive men with a history of previous syphilis was presented by a French group who identified the importance of using VDRL for monitoring of previously treated syphilis in HIV positive patients as TPHA may revert (abstract O207). Interesting studies demonstrating the use of azithromycin in single or repeated doses as a suitable alternative treatment in early syphilis were presented and warrant further investigation (abstracts O208, P595).

\section{Herpes simplex virus (HSV)}

It was interesting to note that the use of aciclovir was being recommended in first episode genital HSV infection in pregnancy although, as Dr L Stanberry (USA) com- mented, its use as suppressive therapy during the last 4 weeks of gestation required further study. Chronic suppressive therapy during pregnancy was not advocated and delegates were reminded to report the use of aciclovir/ valaciclovir to the respective registry. Mention was made of the seroepidemiological studies HSV 2DISC, HSV 2gD plus adjuvant, and HSV $2 \mathrm{gD}$ nucleic acid which are in progress (abstract S9).

\section{Hepatitis}

Data were presented which identified a high prevalence of hepatitis C infection in STD clinic attenders $(3.1 \%$ overall, $4.1 \%$ men and $1.9 \%$ women) raising once again the debate of whether high risk individuals attending STD clinics should be screened and counselled (abstract P449).

\section{Human immunodeficiency virus (HIV)}

The relation between HIV in genital secretions, factors influencing the seminal concentration, STIs and HIV acquisition, and the sexual transmission risks at various stages of the disease were well reviewed by Professor $M$ Cohen (USA) in an excellent plenary lecture. Although antiretroviral therapy may reduce the HIV-RNA concentration in the semen it can still be detected in some individuals. It was hypothesised that there may be the possibility of differential resistance of HIV in the semen. It was felt that monitoring of discordant couples, particularly those on antiretroviral therapy, was necessary (abstract S49).

Professor J Weber (UK), in another scintillating plenary lecture, examined the role CCR5 receptors play in the acquisition of the virus, as incorrect expression or blocking can prevent viral entry, and reviewed the current position of intravaginal virucide development. There has been disappointment in trials with nonoxynol-9, which caused genital inflammation, and failed to show a reduction in HIV transmission (abstract S51). However, the chemical condom appears to be back on the agenda as both PRO 2000 gel and dextran sulphate have performed well on phase 1 trials and are now entering phase 2 trials (abstract $\mathrm{O} 253$ ). Several other agents under consideration are ionophores, non-nucleoside reverse transcriptases, and combination therapy. However, in a study from Mexico of farmers and taxi drivers, the men equated use of vaginal products with infidelity in their wives (abstract P562). Therefore, it may be that the very women who are not empowered to negotiate condom use may also have difficulty in using intravaginal virucides.

\section{Developing countries}

In a precongress workshop we heard that in certain resource poor areas a clinic may be serving over one million individuals. Emphasis was laid on syndromic treatment guidelines which, as Professor AS Latif (Zimbabwe) discussed, should be not only effective but also acceptable, available, accessible, and affordable. However, Dr R C Ballard (South Africa) raised the importance of using surveillance studies to identify varying dis- 
ease patterns and the advantages of molecular techniques in these situations. The need to tailor regimens geographically on the basis of these brought in the debate of adding antiretroviral therapy for genital ulcer disease in areas of high HIV and HSV prevalence.

In the wake of the Mwanza study, which identified a fall in HIV prevalence with syndromic STI treatment, we eagerly awaited details of the Rakai project. This home based mass treatment study, funded by $\mathrm{NIH}$ involves 56 communities in rural Rakai, Uganda, randomised via geographic clusters into intervention or control arms, in order to assess the effect of treatment on the STI prevalence and HIV incidence. Almost 14000 individuals have been recruited, so far. The intervention groups receive mass community treatment with single doses of azithromycin, ciprofloxacin (cefixime if pregnant), and metronidazole, given approximately every 9 months, while the contro groups receive mebendazole (if not pregnant) and vitamin supplements. Over $85 \%$ of sexual contacts reside within a 5 mile area and are presumed to receive treatment by virtue of allocation into the same cluster. Follow up, to date, has shown marked reductions in syphilis, trichomoniasis, gonorrhoea, and $C$ trachomatis. As most of the infected individuals were asymptomatic syndromic management would have failed (abstract S40). Addi- tional advantages seen in this study were of reduced postpartum infection, low birth weight, preterm delivery, and neonatal ophthalmia (abstract S41). HIV infection was seen to reduce the pregnancy rate, as a result of a reduction in conception rate and an increase in spontaneous abortion, hence highlighting the potential for failure if HIV surveillance is targeted only at pregnant women.

New community initiatives are under consideration to improve sexual health and reduce $\mathrm{HIV}$ infection; an interesting one concerning adolescents is planned to run over a 3 year period, and will compare standard sex education with additional peer augmentation and "youth friendly" services (abstracts S43, S44).

\section{Conclusion}

An excellent overview of the current worldwide position of STIs was provided in the congress and new research developments sparkled throughout.

Other highlights included the presentation of the updated 1997 CDC guidelines by Dr K A Workowski (USA), at high speed, although we were pleased to note that we were practising appropriately within United Kingdom licensing laws (abstract S58); the plans described by Dr D Barlow (UK) to develop
pan-European treatment guidelines for genitourinary medicine (abstract S60); the efficacy of counselling interventions for behavioural changes and reductions in new STIs as evidenced by Project RESPECT (abstract O134); the importance of the person time spent in an infectious state (PTI) and the need for improved clinical services, compliance with therapy and health promotion (abstract S11); and the adaptation of mathematical modelling to develop an interactive public health tool enabling the costs and effects of interventions to be studied before implementation (abstract O127).

The achievements of Dr Lawrence Corey and Dr Judith Wasserheit were recognised and both received awards to reflect this. The congress was enjoyed by all and ended on a high note with a genuine flamenco fiesta during which several delegates became dancers!

The next meeting of the ISSTDR will be held on 11-14 July 1999, Denver, Colorado, USA.--Jyoti DHAR, Department of GenitoUrinary Medicine, Derbyshire Royal Infirmary, Derby DE1 2QY and ElizabeTh Carlin, Department of Genito-Urinary Medicine, Kings Mill Centre for Health Care Services, Sutton-inAshfield, Notts, NG17 $4 \mathcal{F H}$.

Abstracts detailed are available in the Congress Abstract Book 